\title{
REGIONALIZACIÓN DE CURVAS DE DURACIÓN DE CAUDALES EN EL DEPARTAMENTO DE ANTIOQUIA-COLOMBIA
}

\author{
SANTiago Gallego ARIAS ${ }^{1}$ \\ Luis Fernando Carvajal Serna ${ }^{2}$
}

\section{RESUMEN}

Los diseños y aprovechamientos en recursos hidráulicos requieren de la información y procesamiento de datos necesario para el éxito de las obras propuestas. Muchas veces los registros de caudal no están disponibles en la cuenca hidrográfica de estudio y uno de los conceptos más usados es la curva de duración de caudales. Este trabajo propone un nuevo acercamiento a la regionalización de la curva de duración de caudales en cuencas sin información. El enfoque presenta una ecuación para cada una de las 6 regiones en que se dividió el departamento de Antioquia, en la parte noroeste de Colombia. La propuesta tiene como variable adicional al enfoque tradicional, la inclusión de la probabilidad de no excedencia. El análisis estadístico realizado muestra que el error de ajuste es menor del 13\% para todas las regiones de Antioquia con excepción de la región de Urabá, que es del 44\%.

PALABRAS CLAVE: Caudales, curva de duración, regionalización de caudales, cuencas hidrográficas con información escasa, Antioquia.

\section{REGIONALIZATION OF FLOW DURATION CURVES IN THE STATE OF ANTIOQUIA, COLOMBIA}

\section{ABSTRACT}

Water resource design and projects need sufficient information and data processes to ensure proposed hydraulic structures are successful. Many hydrological studies have no observed data for discharges in the basins, and one of the most commonly used concepts for previous hydrological studies is the flow duration curve. This work shows a new approach to estimate the flow duration curve in basins for which there is no information. The proposal presents a calibrated equation for each of the six regions in the state of Antioquia, in the northwest of Colombia. Veering from the traditional approach, it includes the non-exceedance probability. The statistical analysis conducted shows a fitting error of less than 13\% for all regions in Antioquia except Urabá, which has an error of 44\%.

KEYWORDS: Discharges, Duration Curve, Discharge Regionalization, Basins with no Gauge Information, Antioquia.

1 Ingeniero Civil. Universidad Nacional de Colombia

2 Ingeniero Civil. Maestría en Recursos Hidráulicos. Universidad Nacional de Colombia

Autor de correspondencia: Carvajal Serna L.F. (Luis Fernando): Facultad de Minas, Cl. 80 \#65 - 223, Medellín, Antioquia, Colombia. Tel.: (574) 4255109

Correo electrónico: Ifcarvaj@unal.edu.co
Historia del artículo:

Artículo recibido: 02-VI-2016 / Aprobado: 18-V-2017

Disponible online: 30 de agosto de 2017

Discusión abierta hasta octubre de 2018 


\section{REGIONALIZAÇÃO DAS CURVAS DE PERMANÊNCIA DE VAZÕES NO DEPARTAMENTO DE ANTIOQUIA, - COLÔMBIA}

\section{RESUMO}

Os desenhos e aproveitamentos em recursos hídricos precisam de informações e processamento de dados necessário para o sucesso das obras propostas. Muitas vezes, os registros de vazões não estão disponíveis na bacia hidrográfica de estudo e um dos conceitos mais utilizados é a curva de duração de fluxo. Este trabalho propõe uma nova aproximação à regionalização da curva de duração de volumes em bacias sem informação. 0 enfoque apresenta uma equação para cada uma das 6 regiões em que se dividiu o departamento de Antioquia, na parte noroeste da Colômbia. A proposta tem como variável adicional ao enfoque tradicional, a inclusão da probabilidade de não excedência. 0 análise estatístic0 realizado mostra que o erro de ajuste é menor de 13\% para todas as regiões de Antioquia com exceção da região de Urabá, que é de $44 \%$.

PALAVRAS-CHAVE: Fluxo, a curva de permanência, racionalização de fluxos, bacias hidrográficas com informações limitadas, Antioquia.

\section{INTRODUCCIÓN}

Colombia es un país que se caracteriza por las fuentes y cursos de agua. Para una adecuada gestión y planificación del recurso hídrico es muy importante el monitoreo de los caudales. Los registros de caudal en una cuenca hidrográfica son una información básica para los estudios hidrológicos. Dependiendo del objetivo del estudio, este incluye caudales medios anuales y mensuales para análisis de oferta y demanda hídrica o análisis energéticos en el largo plazo, y caudales máximos y mínimos para el diseño y operación de obras hidráulicas asociados a un período de retorno (Ven Te Chow et al., 1994).

La calidad de la información hidrológica es de gran importancia. La más confiable es la que proviene de la toma directa de datos, en el caso de los caudales usando estaciones limnimétricas y limnigráficas. Este tipo de fuente de información requiere de la recolección en campo, procesamiento y control de calidad. Aparte de revisar la medición, el procesamiento de los datos y las curvas de calibración, también se hace Análisis de Homogeneidad. En este caso se usan pruebas paramétricas y no paramétri- cas en la media, varianza y tendencia de la serie, Machiwal y Kumar (2012).

El monitoreo de los caudales requiere de redes de medición, las cuales se desearía que tuvieran la mayor cobertura en el área de estudio. La instalación de estaciones de medición tiene como criterios: accesibilidad, costos de operación y mantenimiento.

De otra parte, es común en Colombia el estudio de proyectos de aprovechamiento hidráulico, en los cuales no se dispone de información de caudales. Esta situación agrega incertidumbre a los estudios hidrológicos necesarios para la factibilidad de los proyectos: acueductos, control de inundaciones, riego, centrales hidráulicas, etc. Para solucionar este problema, la hidrología superficial permite reconstruir información de caudales en cuencas hidrográficas donde se carece de registros de caudal. Esta línea de trabajo es lo que se conoce con el nombre de Hidrología con información escasa. La idea principal es transponer mediante métodos generalmente estadísticos, las características de la cuenca con información a la cuenca sin información teniendo presente la coherencia o criterio hidrológico. 
La coherencia hidrológica está representada en el régimen hidrológico, es decir, ambas cuencas deben tener un comportamiento hidrológico similar, precipitación, vientos, condiciones topográficas y usos de los suelos similares, lo que implica que la respuesta de la escorrentía superficial o caudales es similar, Gómez Cano et al. (1990), Guarín y López (1986), Bolaños (1995), Vélez, poveda y Mesa (2000).

Uno de los conceptos de mayor utilidad en la Hidrología es la curva de duración de caudales (CDC). Esta curva se usa para estudios de disponibilidad hídrica, diseño hidráulico, estudio de crecientes, caudales ecológicos y factibilidad de proyectos hidráulicos,. La CDC requiere de constante revisión, tanto en nuevos enfoques de estimación como de actualización de las ecuaciones a medida que se tienen nuevos registros.

Una alternativa que aborda este problema trabaja la CDC como una función exponencial que depende de la probabilidad de no excedencia y se ajustan dos curvas una para una probabilidad menor del 50\% y otra para una probabilidad mayor del 50\%, Vélez, Smith, Pérez, Franco, Bolaños (1995). A nivel nacional, la propuesta más reciente la presenta el Atlas de potencial hidráulico de Colombia, en el cual la curva de duración de caudales (CDC) se estima mediante regionalización ajustando una regresión lineal entre el caudal y el coeficiente de variación para un conjunto de 17 subregiones, UPME-UPJ (2015).

Este trabajo tiene como objetivo proponer un método nuevo de estimación de la CDC en corrientes o ríos del Departamento de Antioquia. Presenta las ecuaciones de regionalización de las CDC para diferentes regiones del departamento, las cuales fueron definidas teniendo en cuenta el régimen hidrológico propio de cada zona.

Este artículo consta de una descripción de la información y del método de la estimación de las CDC con información escasa, ajuste estadístico y ecuaciones de regionalización, análisis de resultados y, por último, conclusiones y referencias bibliográficas.

\section{INFORMACIÓN HIDROLÓGICA Y TOPOGRÁFICA}

Para los estudios encaminados a establecer relaciones entre las variables hidrológicas, el análisis la variabilidad espacial de la precipitación es muy importante, en especial para la determinación del caudal medio de la cuenca. Métodos como el balance hidrológico a largo plazo muestra resultados consistentes en este aspecto, Vélez, Poveda y Mesa (2000).

En Colombia, la variabilidad de la precipitación está definida principalmente por: (1) la situación tropical bajo la influencia de vientos alisios, la oscilación de la Zona de Convergencia Intertropical; (2) sus vecinos Océano Pacífico y Atlántico, siendo fuentes de humedad; (3) la topografía, que incluye la presencia de la cordillera de los Andes, que marcan grandes diferencias climáticas entre los valles interandinos; (4) la circulación propia de la cuenca del Amazonas y (5) la variabilidad de los procesos de la hidrología superficial tales como los contrastes de humedad de suelo y evapotranspiración, fuertemente influenciados por la vegetación, el tipo de suelos y circulación de vientos locales, Mesa, Poveda y Carvajal (1998), Poveda (2004).

Otro aspecto importante es la variación de la precipitación con la altitud. En zonas tropicales como en la que está Colombia es bien conocido el llamado óptimo pluviográfico, el cual corresponde a una elevación en que la precipitación es máxima entre el nivel base y la cima de las cordilleras. La precipitación también se ve afectada por la localización sobre cada uno de los tres ramales Andinos. En cuanto a la cantidad de precipitación en Colombia las zonas más lluviosas están en las vertientes occidental de la cordillera occidental y la vertiente oriental de la cordillera oriental, Vélez et al. (2000). La explicación se da por las circulaciones que se generan en los valles interandinos y en la interacción de los alisios predominantes del este con los vientos que penetran por el oeste, desde el Océano Pacífico, hacia el interior del país. 
En el departamento de Antioquia se pueden identificar tres valles fluviales importantes: al occidente del río Atrato; al centro del estrecho valle del río Cauca; al oriente del gran valle del río Magdalena. Alternándose con los anteriores, se encuentran la Cordillera Occidental entre la depresión del río Atrato y el valle del río Cauca; la Cordillera Central entre el surco del río Cauca y la depresión del Magdalena. La cordillera Occidental está conformada primordialmente por ramales paralelos y transversales al eje principal, mientras que la cordillera central la dominan dos grandes altiplanicies: la de Santa Rosa de Osos al norte y la de Rionegro al sur, estando separadas por el cañón del río Porce, Bolaños (1995). Además lo conforma la zona del bajo Cauca, donde se termina la cordillera Central. Por último la zona del Urabá antioqueño que conecta con las estribaciones de la cordillera Occidental y da salida al departamento hacia el mar Caribe.

Debido a la gran altura de la cordillera Occidental gran parte de las lluvias provenientes del Chocó se precipitan en la ladera occidental, dejando a la ladera oriental con menores precipitaciones. La diferencia de coloración de la vegetación entre la ladera oriental de la cordillera Occidental, más árida, y la ladera occidental de la cordillera Central, más húmeda, es un claro indicador de este proceso, siendo que ambas zonas son apenas separadas por el río Cauca.

La información necesaria para este trabajo es de tres fuentes: precipitación media diaria, el modelo de elevación digital de terreno (MDT) y registros de caudales medios diarios.

El modelo de elevación digital de terreno (MDT) utilizado es obtenido de la misión SRTM (Shuttle Radar Topography Mision). Este MDT tiene un alto grado de pos-procesamiento al ser contrastado con valores reales, y además se apoya en el modelo de la misión ASTER (Advanced Spaceborne Thermal Emission and Reflection Radiometer) de la NASA para la corrección de datos espúreos, ver Figura 1.

Figura 1. Modelo de elevación digital de terreno. Departamento de Antioquia

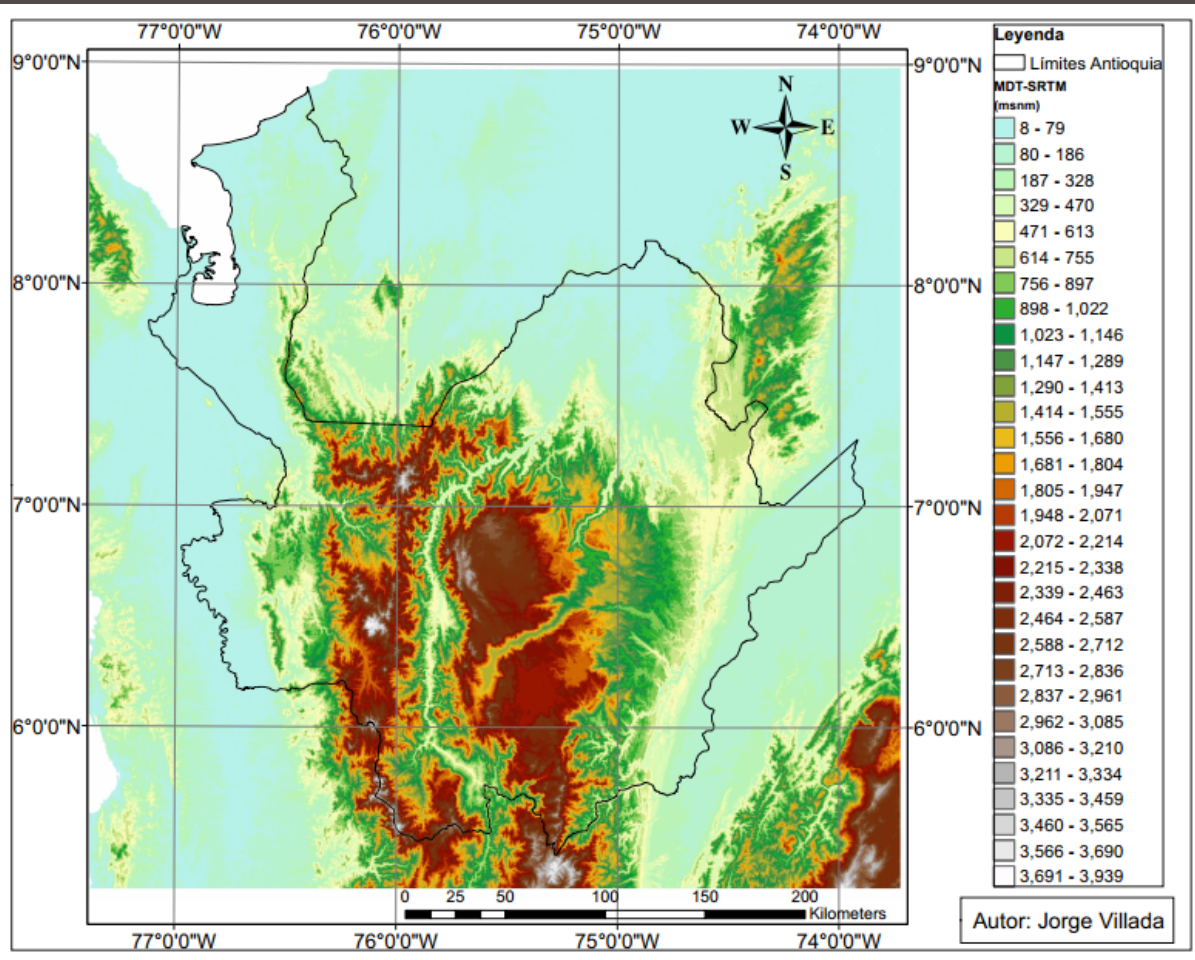


Figura 2. Zonas hidrológicas Departamento de Antioquia y distribución de estaciones de caudal

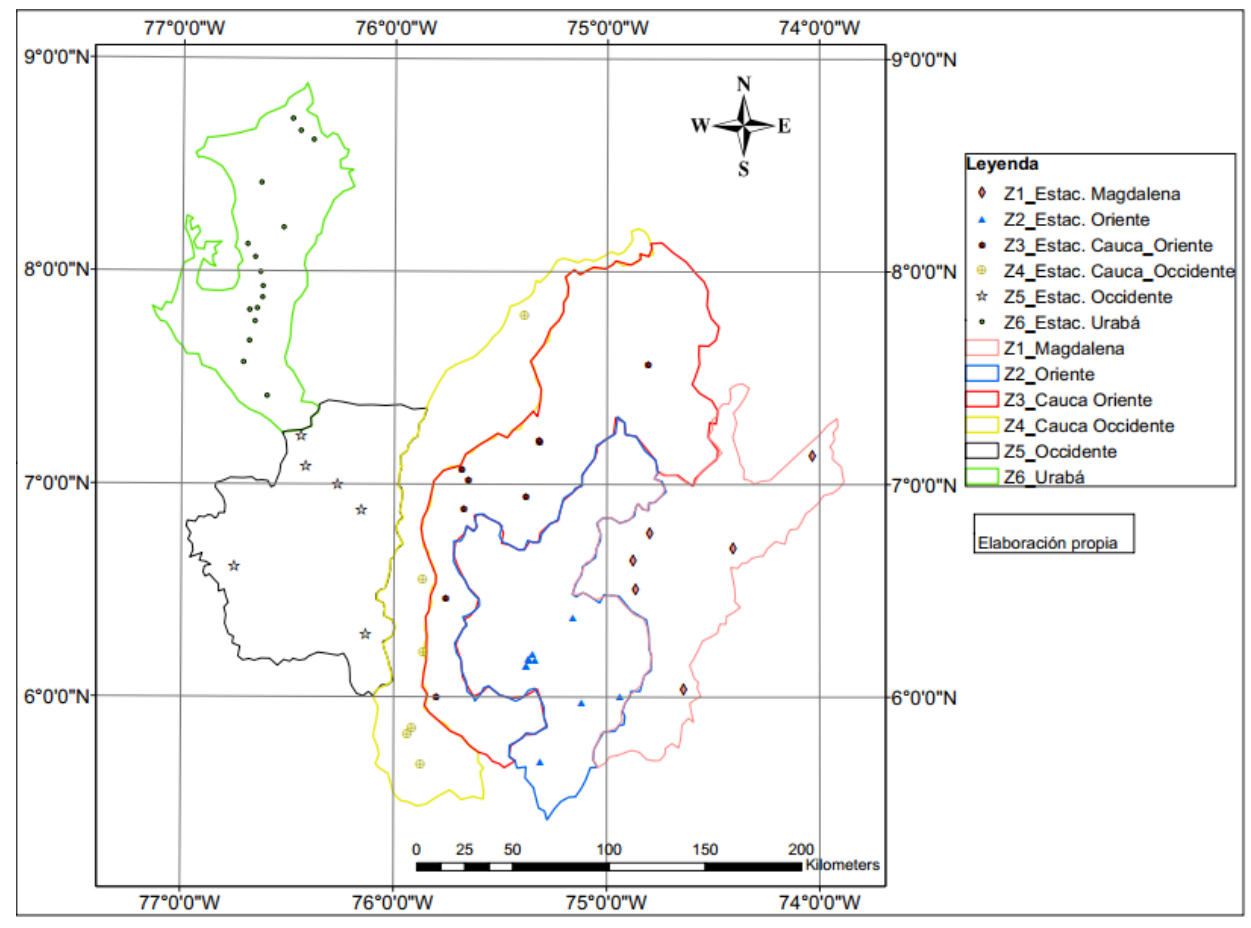

Figura 3. Mapa de precipitación promedia diaria y localización estaciones de precipitación IDEAM y EPM.

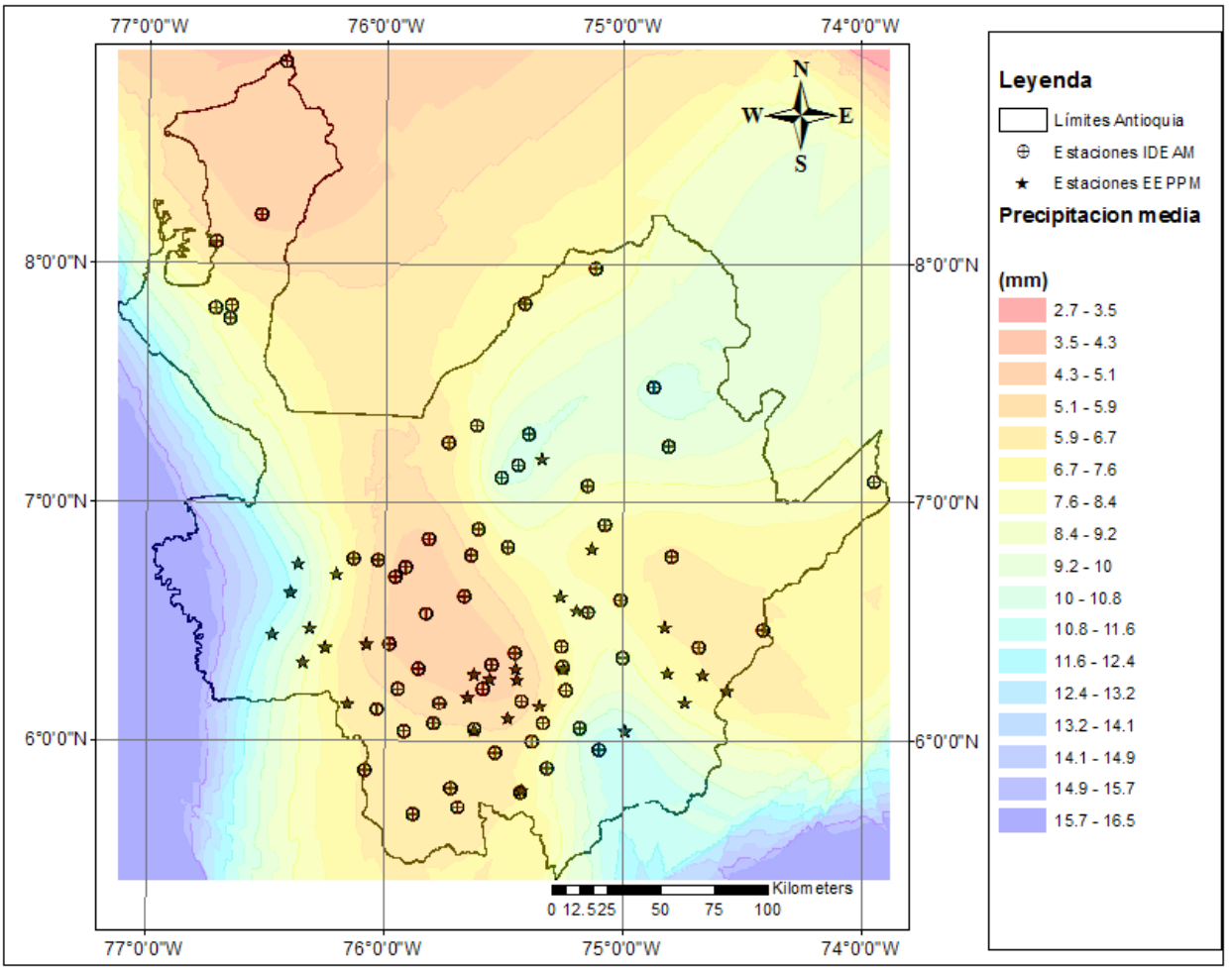


Por otra parte, para este estudio se ha subdividido el departamento de Antioquia en 6 zonas. La información referente a los caudales medios diarios fue suministrada por el Instituto de Estudios Ambientales, IDEAM; se procuró solicitar toda la información posible a esta entidad, lográndose obtener un total de 79 estaciones de caudal medio diario que operan a lo largo y ancho del departamento de Antioquia. Esta información se sometió a un proceso de selección, teniendo en cuenta criterios como: la cantidad de registros neta debe ser superior a 10 años, además los registros de los ríos Atrato, Cauca y Magdalena también se depuraron del análisis, pues se consideró que ellos tenían un comportamiento hidrológico dependiente de factores externos a los que dominan la zona antioqueña.

La Figura 2 muestra la subdivisión del departamento en las zonas hidrológicas junto con la distribución de las estaciones de caudal finalmente seleccionadas.

La información de precipitación mensual comprende un total de 61 estaciones dentro del departamento de Antioquia operadas por el IDEAM. Para complementar aquellas zonas que carecían de información de precipitación, se utilizó la información presentada en el trabajo de Smith y Vélez, 1998. Esta información corresponde a 29 estaciones operadas por Empresas Públicas de Medellín.

El mapa de precipitación promedia diaria se obtuvo a partir de la técnica de Krigging con deriva externa, en esta caso, las cotas del MDT, apoyado en el software ArcMap 10. La Figura 3, presenta la localización de las estaciones de precipitación en Antioquia y el mapa de precipitación resultante de la interpolación por Krigging.

\section{ESTIMACIÓN CURVAS DE DURACIÓN DE CAUDALES REGIONALIZADAS}

La CDC es una herramienta utilizada para indicar la distribución de la ocurrencia de niveles de caudales a lo largo del tiempo (Guarín y López, 1986). Para su elaboración se ordenan los valores de caudales registrados de mayor a menor y se asigna a cada valor de caudal la probabilidad de excedencia (función de probabilidad empírica Weibull). Luego, se grafican los datos de caudal en el eje de las ordenadas y los respectivos valores de probabilidad en el eje de las abscisas.

$$
F E(x)=\frac{m}{N+1}
$$

Donde:

$F E(x)=$ probabilidad de excedencia del valor " $\mathrm{x}$ ".

$\mathrm{m}=$ posición en la columna ordenada de mayor a menor del valor " $\mathrm{x}$ ".

$\mathrm{N}=$ número de total de datos.

Para este trabajo se realizó una regionalización de las curvas de duración de caudal basándose en la homogeneidad hidrológica de una zona, teniendo en cuenta la topografía y régimen de lluvia. Siguiendo como base el procedimiento de regionalización de características medias de caudal presentado en Smith y Vélez, 1997, el procedimiento desarrollado es el siguiente:

a) Se seleccionaron aquellas estaciones de caudal que presentan un registro neto equivalente mayor a diez años. A cada una de estas series se le construye la CDC. Una longitud mínima de 10 años obedece a tener al menos un fenómeno El NinoOscilación del Sur, y descartar un número mayor de estaciones de caudal.

b) Se definen aquellas zonas que pueden tener un comportamiento hidrológico similar; inicialmente se tuvo en cuenta la división realizada por Jaramillo y Chávez, 2000. La división tuvo en cuenta la distribución temporal de la lluvia, además de la precipitación total y la ubicación geográfica.

c) Se grafican para cada zona los caudales medios diarios de cada estación vs los caudales de los diferentes percentiles o probabilidades de no excedencia de la CDC.

d) Para cada zona, se planteó el ajuste de una ecuación de la forma: 


$$
Q_{p}=\frac{A Q_{m}+B}{D \frac{P_{q}}{C}}
$$

Donde:

$Q_{m}:$ es el caudal medio diario de la cuenca en $\mathrm{m}^{3} / \mathrm{s}$.

$A, B, C$ y $D$ son parámetros.

$P_{q}$ : es la probabilidad de no excedencia en la CDC en porcentaje.

$Q_{p}$ : es el valor del caudal de probabilidad de no excedencia en $\mathrm{m}^{3} / \mathrm{s}$.

La Ecuación 2, permite utilizar una sola expresión para cada zona con sólo tener el caudal medio y reemplazar $P_{q}$ por el valor del porcentaje del tiempo del caudal que se desea calcular o probabilidad de no excedencia. La Ecuación 2, se sugiere por la relación que se observa al graficar los valores de los caudales medios diarios contra los valores de los caudales de diferentes probabilidades de no excedencia, $Q_{m} v s Q_{p}$. La Figura 4, muestra la relación para la zona 3 de los caudales con una probabilidad de no excedencia de 30, 50 y $70 \%$ de cada cuenca.
Como se observa en la Ecuación 2, es necesario conocer el caudal medio diario de la cuenca, el cual se puede estimar por diferentes metodologías, como la de rendimiento, la cual se basa en una proporcionalidad de áreas, Gómez Cano et al. (1990), o la ecuación del balance hídrico, Vélez et al. (2000). La ecuación de balance hídrico de largo plazo se expresa de la siguiente manera:

$$
Q_{m}=(P-E) A
$$

Donde:

$Q_{m}$ : es el caudal medio anual en $\mathrm{m}^{3} / \mathrm{s}$.

$P:$ es la precipitación media anual en lámina $\mathrm{mm} /$ día.

$E$ : es la evapotranspiración media anual en lámina en mm/día. Para la estimación de la evapotranspiración se utiliza la ecuación de Cenicafé combinada con la ecuación de Budyko, Vélez et al. (2000).

$A$ : es el área de la cuenca en $\mathrm{m}^{2}$.

Como alternativa para el cálculo del caudal medio diario se propone una ecuación de regionalización del caudal en función de parámetros geomorfológicos. El procedimiento es el siguiente:

Figura 4. Qm vs Qp, para diferentes probabilidades de no excedencia en la zona 3, afluentes vertiente oriental de la cuenca del río del Cauca-Departamento de Antioquia

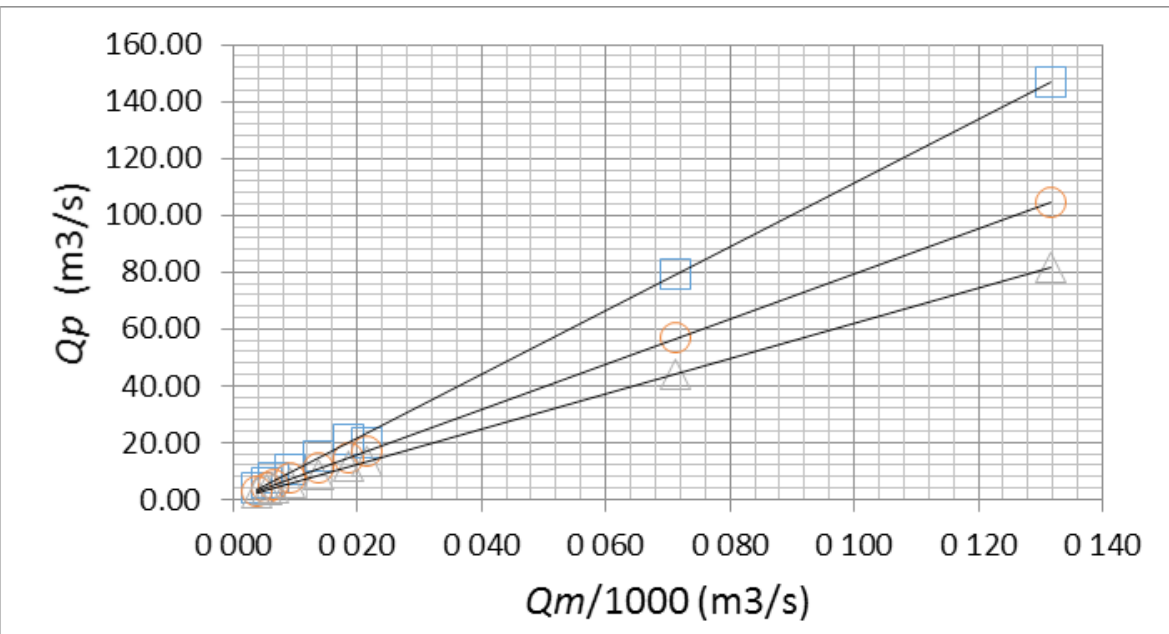


a) Se miden los parámetros geomorfológicos para las cuencas definidas por la estación de aforo como el área, el perímetro, la pendiente del cauce principal, la pendiente promedio de la cuenca, además de la precipitación promedio diaria multianual.

b) Con las variables geomorfológicas y climáticas medidas se ajusta la expresión 4, para establecer el caudal medio, $Q_{m^{\prime}}$ de las cuencas:

$$
Q_{m}=K A^{\alpha} S p p l^{\theta} S c^{\delta} K c^{\mu} P m^{\beta}
$$

Donde:

$K, \alpha, \theta, \delta, \mu \mathrm{y} \beta$ son parámetros encontrados en la regresión.

A es el área de cuenca en $\mathrm{Km}^{2}$.

Sppl es la pendiente promedio del cauce principal en \%.

$S c$ es la pendiente promedio de la cuenca en $\%$.

$P m$ es la precipitación media diaria en la cuenca en mm/día.

$Q m$ es el caudal medio diario de la cuenca en $\mathrm{m}^{3} / \mathrm{s}$.

$K c$ es el coeficiente de Gravelius, la relación entre el perímetro de la cuenca y el perímetro de un círculo de igual área que la cuenca:

$$
K c=0,28 \frac{P e r}{\sqrt{A}}
$$

Donde:

Per es el perímetro de la cuenca en km.

$A$ es el área de cuenca en $\mathrm{Km}^{2}$.

Este método supone que la zona a la que se quiere determinar el caudal medio diario tiene características geomorfológicas y climatológicas similares de acuerdo a las regiones definidas en el departamento de Antioquia.

Para la estimación del error de ajuste de las curvas de duración se usó el promedio del error relativo porcentual en valor absoluto, definido como:

$$
E \bar{r} r \%=\frac{1}{\mathrm{~N}} \sum_{i=1}^{\mathrm{N}} \frac{\left|O_{i}-E_{i}\right|}{O_{i}} \times 100
$$

Donde:

$E$ : es el valor del caudal estimado en $\mathrm{m}^{3} / \mathrm{s}$.

$O$ : es el valor del caudal observado en $\mathrm{m}^{3} / \mathrm{s}$.

$N$ : el número de datos.

$E \bar{r} r \%$ : es el promedio error relativo porcentual.

\section{RESULTADOS}

A continuación se presentan las ecuaciones para la curva de duración de caudal regionalizada hallada para cada una de las zonas. Estas curvas se han ajustado para el rango de $10 \%$ a $90 \%$ de duración del caudal o probabilidad de no excedencia.

Zona 1: Zona Magdalena

$$
Q \%=\frac{27.048 Q m+5.092}{10.47+\frac{P q}{2.064}}, E \bar{r} r \%=13.15 \%
$$

Zona 2: Zona Oriente

$$
Q \%=\frac{26.7 Q m}{10.494+\frac{P q}{2.07}}, E \bar{r} r \%=10.85 \%
$$

Zona 3: Zona Cauca oriente

$$
Q \%=\frac{28.217 Q m}{10.031+\frac{P q}{1.97}}, E \bar{r} r \%=9.68 \%
$$

Zona 4: Zona Cauca occidente

$$
Q \%=\frac{27.621 Q m+1.88}{10.345+\frac{P q}{2}}, \ldots E \bar{r} r \%=12.84 \%
$$

Zona 5: Zona occidente

$$
Q \%=\frac{28.424 Q m}{10,452+\frac{P q}{2.143}}, . . E \bar{r} r \%=8.16 \%
$$


Zona 6: Zona Urabá

$$
Q \%=\frac{3.834 Q m}{-0.8+\frac{P q}{3.97}}, E \bar{r} r \%=44.05 \%
$$

El otro aspecto a calcular es el caudal medio diario, el cual se puede determinar con las ecuaciones 13 a 18. Se debe aclarar que el error típico en algunas zonas (Magdalena Oriente, Occidente río Cauca y Suroccidente) no se calcula porque en la regresión se utilizan 5 tipos de variables $(A, S p p l, S c$, $K c$ y $P m$ ) y en aquellos lugares sólo se contaba con un total de 5 estaciones, por lo que en teoría el error es cero.

\section{Zona 1: Zona Magdalena}

$$
\begin{gathered}
Q_{m}=0.4763 A^{1.0047} S_{p p l}^{1.4579} S_{c}^{-3.8923} K_{C}^{-2.0933} \\
P_{m}^{5.5282}, \text { error }=---\%
\end{gathered}
$$

Zona 2: Zona oriente

$$
\begin{gathered}
Q_{m}=0.00575 A^{1.208} S_{p p l}^{0,4109} S_{c}^{-0.696} K_{C}^{1.441} \\
P_{m}^{1.4087}, \text { error }=29 \%
\end{gathered}
$$

Zona 3: Zona Cauca oriente

$$
\begin{gathered}
Q_{m}=0.00027 A^{0.9815} S_{p p l}^{0.1934} S_{c}^{0.2573} K_{C}^{1.2561} \\
P_{m}^{1.6578} \text {, error }=14,8 \%
\end{gathered}
$$

Zona 4 Vertiente Cauca occidente

$$
\begin{gathered}
Q_{m}=8.3 \times 10^{-12} A^{1.4532} S_{p p l}^{3.1542} S_{c}^{1.5184} K_{C}^{8.8151} \\
P_{m}^{2.6258} \text {, error }=---\%
\end{gathered}
$$

Zona 5: Zona occidente

$$
\begin{gathered}
Q_{m}=40.0395 A^{0.9292} S_{p p l}^{-0.7731} S_{c}^{-1.0114} K_{C}^{1.764} \\
P_{m}^{-1.1572}, \text { error }=---\%
\end{gathered}
$$

Zona 6: Zona Urabá

$$
\begin{gathered}
Q_{m}=0.0165 A^{1.3284} S_{p p l}^{1.0472} S_{c}^{-1.7516} K_{C}^{-1.5558} \\
P_{m}^{2.5213} \text {, error }=28.7 \%
\end{gathered}
$$

\section{CONCLUSIONES}

Se ha llegado a una serie de ecuaciones útiles para la ayuda en la evaluación preliminar de proyectos hidroeléctricos en el departamento antioqueño, principalmente su utilidad será en aquellas zonas sin información de caudales.

La Zona 6, región Urabá presenta los mayores porcentajes de error; entre otras dificultades de ajuste, es preciso que se realice un proceso de verificación de validez de la información.

En Hidrología los análisis de regionalización permiten estimar la variable hidrológica de interés en áreas, sin información, lo que permite ganar en espacialidad en los resultados y análisis, pero se debe tener en cuenta la incertidumbre en estos métodos en cuencas en zonas intertropicales que no distan mucho entre sí, por la alta variabilidad, especialmente de los procesos convectivos en cuencas hidrográficas.

En futuros estudios tener en cuenta una mayor red de medición, considernado información de caudales de otras entidades. Información adicional permite mejorar la estimación de los parámetros de las ecuaciones.

Los resultados obtenidos son a nivel de prefactibilidad y no exoneran de realizar estudios detallados para cada proyecto en particular y tomar las decisiones más adecuadas para el mismo.

Las ecuaciones de regionalización presentadas en este artículo, muestran un ajuste adecuado para un rango de probabilidad de excedencia entre el $10 \%$ y $90 \%$. Por fuera de este rango se encontró que para la estimación de valores extremos, el error calculado aumenta fuertemente, indicando grandes diferencias hidrológicas.

\section{REFERENCIAS}

Bolaños Arias, H. D. Regionalización de caudales mínimos en el departamento de Antioquia. Trabajo de Grado, Universidad Nacional de Colombia, Sede Medellín, 1995. $150 \mathrm{p}$.

Gómez Cano, G. I., Piedrahita Moreno, J. J., Rojas Monsalve, J. J. y Sanz Henao, J. A. Metodología para el inventario de centrales hidroeléctricas de mediana capacidad entre 10 y 100 MW y su aplicación a una cuenca específica. Trabajo de Grado, Universidad Nacional de Colombia. 1990. 244 p. 
Guarín Flórez, N. de J., y López Ospina, G. V. Regionalización del comportamiento esperado de estructuras hidráulicas de derivación operadas a filo de agua. Trabajo de Grado, Universidad Nacional de Colombia, 1986. 189 p.

Jaramillo-Robledo, Á., \& Chaves-Córdoba, B. (2000). Distribución de la precipitación en Colombia analizada mediante conglomeración estadística. Cenicafé, 51(2), pp. 102-113.

Machiwal, D. y Kumar, M. Hydrological Time Series Analysis-Theory and Practice. Ed. Springer, 2012.316 p.

Poveda, Germán; Mesa, Oscar y Carvajal, Luis F. Introducción al clima de Colombia. Editorial Universidad Nacional de Colombia, 1998. 390 p.

Poveda, Germán (2004). The hydro-climatology of Colombia: a synthesis from inter-decadal to diurnal timescales (in Spanish). Rev Academia Colombiana de Ciencias, 28(107), pp. 201-222.

Smith Quintero, Ricardo y Vélez Otálvaro, María V. Hidrología de Antioquia. Universidad Nacional de Colombia y Secretaría de Obras Públicas de Antioquia, Medellín, 1997, 163 p.

UPME-PPUJ. Atlas potencial hidroenergético de Colombia. 2015. 160 p.

Vélez, María V., Smith, Ricardo, Pérez, Carlos A., Franco, Carlos Jaime y Bolaños, Hernán (1995). Aplicación de algunas técnicas al diseño hidrológico con información escasa. Revista Avances en Recursos Hidráulicos, Posgrado en Aprovechamiento de Recursos Hidráulicos, núm. 3, pp. 35-54.

Ven Te, Chow, Maidment, David y Mays, Larry. Hidrología Aplicada. Ed. MacGraw-Hill, 1994, 582 p.

Vélez, Jaime I., Poveda, Germán y Mesa, Oscar. Balances hidrológicos de Colombia. Medellín: Universidad Nacional de Colombia, 2000.150 p.

PARA CITAR ESTE ARTÍCULO TO REFERENCE THIS ARTICLE / PARA CITAR ESTE ARTIGO /

Gallego Arias, S.; Carvajal Serna, L.F. (2017). Regionalización de curvas de duración de caudales en el departamento de Antioquia-Colombia. Revista EIA, 14(27), enero-junio, pp. 21-36. [Online]. Disponible en: https://doi.org/10.24050/ reia.v14i27.1158 